\title{
An Adaptive Neuro Fuzzy Inference System for Fault Detection in Transformers by Analyzing Dissolved Gases
}

\author{
Ms. Alamuru Vani. ${ }^{\mathrm{a},}$ Dr. Pessapaty Sree Rama Chandra Murthy. ${ }^{\mathrm{b}}$ \\ ${ }^{a}$ Associate Professor, Department of Electrical Engineering, VJIT, Hyderabad, \\ ${ }^{b}$ Dean, School of Electrical Engineering, Sreenidhi Institute of Science and Technology, Hyderabad
}

\begin{abstract}
Safe operation of elements of power systems plays a crucial role in maintaining the reliability and safety of the system. Transformers being a key element in power systems need to be maintained and monitored on a regular basis. Dissolved gas analysis has been used as a reliable tool in maintaining the safe operation of transformers for a long time. Analysis of dissolved gases is analytical and often interpreted differently by different users and methods. The scope of Artificial Intelligence tools in dissolved gas analysis has become critical with increasing number of transformers being used in power systems coupled with rapid expansion of transmission and distribution components. In this work we have designed an analysis system based on different Artificial Intelligence methods like Neural Networks, Fuzzy, and Adaptive Neuro-Fuzzy for analyzing dissolved gas and give interpretation about possible faults. Adaptive Neuro-Fuzzy Inference System (ANFIS) modeling technique has emerged as one of the soft computing modeling technique for power transformer. The objective of this paper is to design an ANFIS model for dissolved gas analysis of power transformers. The prediction ability of the ANFIS is also tested using limited data set for model training.
\end{abstract}

Keywords: Artificial Intelligence, Neural Networks, Neuro-Fuzzy.

\section{Introduction}

Dissolved Gas Analysis (DGA) has been used for more than 30 years [1]-[3] for the condition assessment of functioning electrical transformers. DGA measures the concentrations of hydrogen $(\mathrm{H} 2)$, methane $(\mathrm{CH} 4)$, ethane $(\mathrm{C} 2 \mathrm{H} 6)$, ethylene $(\mathrm{C} 2 \mathrm{H} 4)$, acetylene $(\mathrm{C} 2 \mathrm{H} 2)$, carbon monoxide $(\mathrm{CO})$ and carbon dioxide $(\mathrm{CO} 2)$ dissolved in transformer oil. $\mathrm{CO}$ and $\mathrm{CO} 2$ are generally associated with the decomposition of cellulose insulation; usually, small amounts of $\mathrm{H} 2$ and $\mathrm{CH} 4$ would be expected as well. $\mathrm{C} 2 \mathrm{H} 6, \mathrm{C} 2 \mathrm{H} 4, \mathrm{C} 2 \mathrm{H} 2$ and larger amounts of $\mathrm{H} 2$ and $\mathrm{CH} 4$ are generally associated with the decomposition of oil. All transformers generate some gas during normal operation, but it has become generally accepted that gas generation, above and beyond that observed in normally operating transformers, is due to faults that lead to local overheating or to points of excessive electrical stress that result in discharges or arcing. Despite the fact that DGA has been used for several decades and is a common diagnostic technique for transformers, there are no universally accepted means for interpreting DGA results IEEE C57-104 [3] and IEC 60599 [4] use threshold values for gas levels. Other methods make use of ratios of gas concentrations [2], [5] and are based on observations that relative gas amounts show some correlation with the type, the location and the severity of the fault. Gas ratio methods allow for some level of problem diagnosis whereas threshold methods focus more on discriminating between normal and abnormal behavior. The IEC standard 60599 [18] classifies the DGA detectable transformer faults into 2 categories: the electrical fault and the thermal fault. These two main categories can be further sorted into 6 types of transformer fault, according to the magnitudes of the fault energy: the electrical fault: partial discharge (PD ), D1 (discharge of low energy) and D2 (discharge of high energy); the thermal fault: T1 (Thermal fault of low temperature range, $\mathrm{T}<300{ }^{\circ} \mathrm{C}$ ), $\mathrm{T} 2$ (Thermal fault of medium temperature range, $300{ }^{\circ} \mathrm{C}<\mathrm{T}<700{ }^{\circ} \mathrm{C}$ ) and $\mathrm{T} 3$ (Thermal fault of high temperature range, $\mathrm{T}>700^{\circ} \mathrm{C}$ ) [7].

Many DGA analysis techniques employing Artificial Intelligence can be found in the literature. We briefly review here previous techniques for transformer failure prediction from DGA. All of them follow the methodology consisting in feature extraction from DGA, followed by a classification algorithm. The majority of them are techniques [6], [7], [9]-[13], [15], [16] built around a feed-forward neural-network classifier, that is also called Multi-Layer Perceptron (MLP) and that we explain in Section IV. Some of these papers introduce further enhancements to the MLP: in particular, neural networks that are run in parallel to an expert system in [10], Wavelet Networks (i.e. neural nets with a wavelet-based feature extraction) in [16], Self-Organizing Polynomial Networks in [9] and Fuzzy Networks in [6], [12], [13], [15]. Several studies [6], [8], [12], [13], [15], [16] resort to fuzzy logic [18] when modeling the decision functions. Fuzzy logic enables logical reasoning with continuously-valued predicates (between 0 and 1) instead of binary ones, but this inclusion of uncertainty within the decision function is redundant with the probability theory behind Bayesian reasoning and statistics. Stochastic optimization techniques such as genetic programming are also used as an additional tool to select features for the classifier in [8], [12], [14], [16], and [17]. Finally, Shintemirov et al. [17] conduct a 
comprehensive comparison between k-nearest neighbors, neural networks and support vector machines each of them combined with genetic Programming-based feature selection.

This work elaborates on a tool that incorporates Artificial Intelligence methods for making a comprehensive analysis of dissolved gases in transformer oil. The primary motivation for the work is to provide a platform for analysis of dissolved gases to help in the early detection and diagnosis of transformer faults. The idea is to provide the user with a wide variety of options in analyzing a fuzzy situation which may arise because of different interpretations that shall be provided by different traditional analysis methods. We hope that such a tool will go a long way in detection and diagnosis of transformer faults by minimizing human interpretation errors. This work is carried out with assistance from with Andhra Pradesh State Transmission Corporation (APTRANSCO) in the form of required transformer analysis data and expert opinion for validation of the tool.

\section{Problem Statement}

The Dissolved Gas Analysis is a diagnostic and maintenance tool used in machinery. Through this method, gases are studied to give an early indication of transformer abnormal behavior. For the last 20 years, this method is widely used for detecting and diagnosing the incipient faults of power transformers. Its effectiveness has been proven by a lot of well known electrical testing laboratories or institutions such as The Institute of Electrical and Electronics Engineers (IEEE), Central Electricity Generating Board of Great Britain (CEGB), International Electro technical Commission (IEC), etc.. Today, numbers of diagnostic methods based on the DGA have been proposed by researchers in the power transmission field from all over the world.

The aim of the proposed work is to design a comprehensive tool for dissolved gas analysis that incorporate artificial intelligence elements to aid in incipient transformer fault detection. The objectives of the proposed tool can be listed as follows, to provide seamless integration between different methods of analysis by enabling flexible and easy use of the tool; use hybrid artificial intelligence elements like Neural Network, Fuzzy and Adaptive Neuro -Fuzzy methods to improve the diagnostic accuracy of the tool; provide the user with wide variety of options that include traditional methods of analysis like Rogers Ratio, IEC, Duval Triangle etc... to provide a holistic approach in analyzing transformer faults.

\section{A Fuzzy Approach for Dissolved Gas Analysis}

Fuzzy logic had been applied in various fields such as control system, decision support, fault diagnostics, image processing and data analysis. The fuzzy logic theory was applied in solving nonlinear control problems heuristically and modularly along linguistic lines. The advantages of fuzzy logic are that it exhibits the nature of human thinking and makes decision or judgment using linguistic interpretation. Furthermore, the control rules, regulations and methods based on the perception, experience and suggestion of a human expert were encoded in the meaningful way to avoid mathematical modeling problems.

\subsection{Fuzzy Rogers Ratio}

Rogers Ratio method uses the 4-digit ratio code generated from the 5 fault gases which are Acetylene, Ethylene, Methane, Hydrogen and Ethane to determine 15 transformer conditions. Therefore, the structure for the Fuzzy Rogers Ratio is such that the four ratio codes are identified as the input parameter while the 15 interpretation results based on the difference combination of ratio code are identified as the output parameter. The approach used in fuzzifying the gas ratios according to the method of Roger's Ratio is discussed here.. The real variables are converted into the appropriate linguistic variables. The 4 ratios are classified as Low (Lo), Medium (Med), High (Hi) and Very High (Vhi) term set according to their membership intervals as defined below:

$\mathrm{AE}=\{\mathrm{Lo}, \mathrm{Med}, \mathrm{Hi}\}$

$\mathrm{MH}=\{\mathrm{Lo}, \mathrm{Med}, \mathrm{Hi}, \mathrm{Vhi}\}$

$\mathrm{EE}=\{\mathrm{Lo}, \mathrm{Med}, \mathrm{Hi}\}$

$\mathrm{EM}=\{\mathrm{Lo}, \mathrm{Hi}\}$

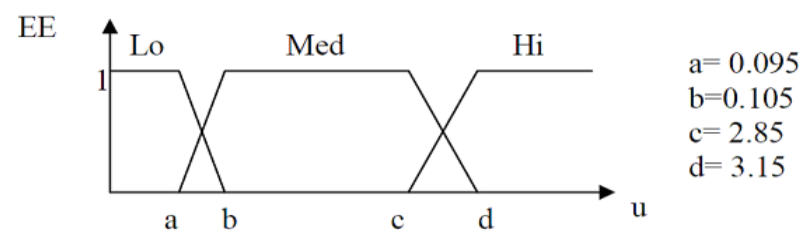

Figure (1): Structure of Membership Function used for Ethylene / Ethane (EE) 
Fuzzy inference rules consist of a collection of rules which are extracted from the expert. Normally, fuzzy inference consists of two components which are antecedent (if part) and consequent (then part). For this application, the fuzzy inference rules can be extracted from the Roger's ratio fault interpretation guide. There are a total of 22 fuzzy inference rules that can be derived from Rogers fault interpretation. However, with the fuzzy logic techniques which allow partial membership may improve the number of matched cases as compared to the ordinary crisp set theory. The output of the fuzzy inference can be obtained using the Mamdani's Max-Min composition technique.

\subsection{Fuzzy Key Gas Method}

A set of rules to diagnose abnormalities such as Thermal, Corona or Arcing problems is employed The Key Gas method. It is a reliable diagnostic method because it can be used to diagnose the condition of the transformer even there are only a few gases obtained from the oil sample. Comparatively, the Rogers Ratio method requires all 5 necessary ratio gases to be detected correctly earlier to produce satisfactory result. However, there is a possibility that the ratio code cannot provide meaningful information due to the absent of certain gases. In this case, Fuzzy Key Gas method which uses the individual gas rather than the calculation gas ratio for detecting fault condition will be a perfect candidate to offset the limitation of the Rogers Ratio method. The quantization step is to define the threshold values for all the 7 input gases. The international recognized standard can be used to define the threshold value for Key Gas method. Based on the IEEE Standard, 7 input variables have been classified into Low (Lo), Medium (Med) and High (Hi) term set. From the 3 term sets, the IEEE standard value is being used as the medium term set while the high and low term set are being adjusted 5 percent more or 5 percent less than the medium term set respectively. For the Fuzzy Key Gas fault diagnostic method, the appropriate types of membership function are Triangular, L-function and $\Gamma$-function. The fuzzy membership function for the Key Gas input for $\mathrm{H} 2, \mathrm{CO}, \mathrm{CO} 2, \mathrm{C} 2 \mathrm{H} 2, \mathrm{C} 2 \mathrm{H} 4, \mathrm{C} 2 \mathrm{H} 6, \mathrm{CH} 4$ and the Figure (2) depicts the structure of membership function used for Carbon Monoxide (CO)

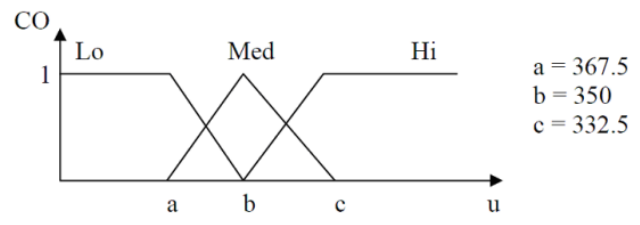

Figure (2): Structure of Membership Function used for CO

The output of the fuzzy inference can be obtained using the Mamdani's Max-Min composition .The consequent is computed as follows:

Corona $(\mathrm{CN})=\operatorname{Max}\{$ Rule 1$\}$

Cellulose Insulation Breakdown $(\mathrm{CIB})=$ Max $\{$ Rule 4, Rule 5, Rule 7, Rule 10$\}$

Low Temperature Oil Breakdown $($ LTOB) $=$ Max $\{$ Rule 19, Rule 20, Rule 21, Rule 22, Rule 25$\}$

High Temperature Oil Breakdown $(\mathrm{HTOB})=\operatorname{Max}\{$ Rule 16 $\}$

Arcing $(\mathrm{ARC})=\operatorname{Max}\{$ Rule 13$\}$

A suitable defuzzification method for fuzzy diagnosis system is the Max-membership defuzzification method where the element that has the maximum degree of membership function is chosen is used.

\section{ANN Based System for Transformer Incipient Fault Diagnosis}

The basic idea of neural network based diagnosis is non-linear mapping input and outputs. Both back propagation network (BPN) and probabilistic neural network (PNN) are used to diagnose the transformer faults in its incipient stage. An artificial neural network (ANN) includes selection of inputs, outputs, network topology and weighed connection of node. Input features will correctly reflect the characteristics of the problem [18]. Another major work of the ANN design is to choose network topology. This is done experimentally through a repeated process to optimize the number of hidden layers and nodes according to training and prediction accuracy. In this work 7 key gases namely $\mathrm{H} 2, \mathrm{CO}, \mathrm{CO}_{2}, \mathrm{C}_{2} \mathrm{H}_{2}, \mathrm{C}_{2} \mathrm{H}_{4}, \mathrm{C}_{2} \mathrm{H}_{6}$, and $\mathrm{CH}_{4}$ are analyzed to diagnose 5 different fault conditions namely, Corona $(\mathrm{CN})$, Cellulose Insulation Breakdown (CIB), Arcing (ARC), Low Temperature Oil Breakdown (LTOB) and High Temperature Oil Breakdown (HTOB).In this work a Feed Forward Back Propagation network is used. A TRAINLM training function along with LEANGDM adaptive learning function is used of training and adaptation of the network. MSE is used to compute the performance measure. The total network comprises of 2 layers with layer one having 10 neurons and using a TANSIG transfer function. The regression plot of the regression plot of the network used in the work is given in the Figure (1) 

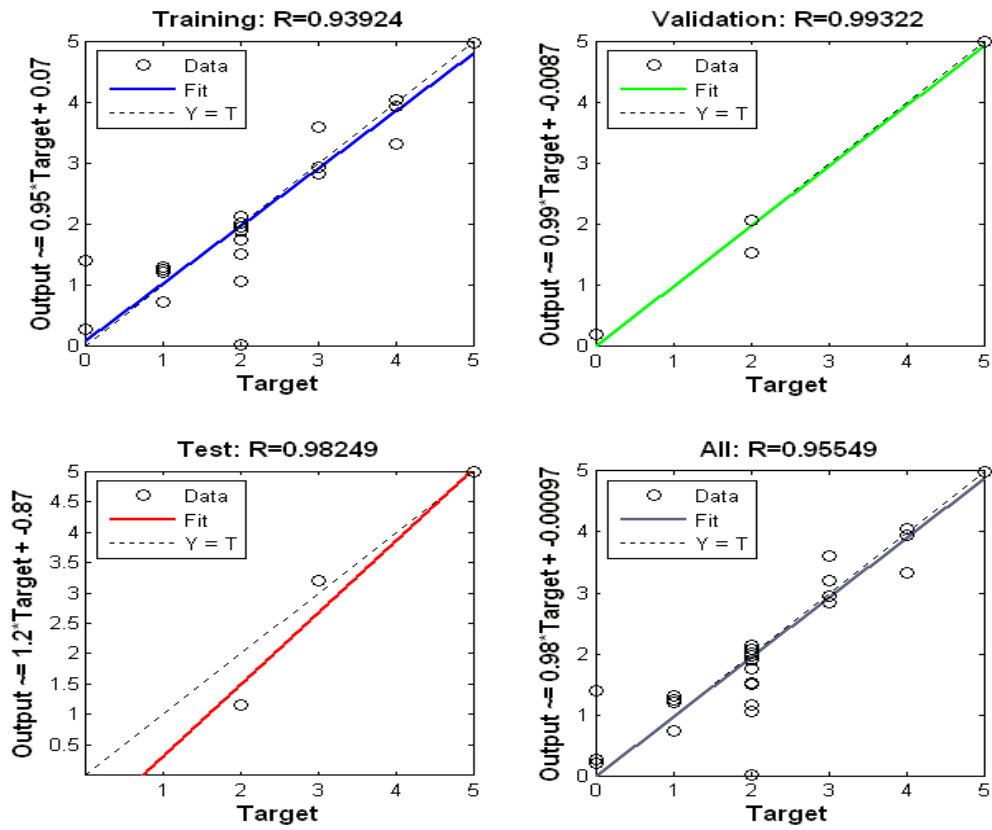

Figure (3) Regression plot of the ANN used in Analysis

\section{Adaptive Neuro Fuzzy Inference System}

Adaptive Neuro-Fuzzy Inference System (ANFIS) is a class of adaptive networks that is functionally equivalent to fuzzy inference system. Sugeno type ANFIS [19] uses a hybrid learning algorithm to identify parameters of Sugeno-type fuzzy inference system. It applies a combination of the least squares method and the back propagation gradient descent method for training FIS membership function parameters to emulate a given training data set. An ANFIS works [20] by applying neural learning rules to identify and tune the parameters and structure of a Fuzzy Inference System (FIS). There are several features of the ANFIS which enable it to achieve great success in a wide range of scientific applications. The attractive features of an ANFIS include: easy to implement, fast and accurate learning, strong generalization abilities, excellent explanation facilities through fuzzy rules, and easy to incorporate both linguistic and numeric knowledge for problem solving. According to the neuro-fuzzy approach, a neural network is proposed to implement the fuzzy system, so that structure and parameter identification of the fuzzy rule base are accomplished by defining, adapting and optimizing the topology and the parameters of the corresponding neuro-fuzzy network. The network can be regarded both as an adaptive fuzzy inference system with the capability of learning fuzzy rules from data, and as a connectionist architecture provided with linguistic meaning. The $\mathrm{H}_{2}, \mathrm{CH}_{4}, \mathrm{C}_{2} \mathrm{H}_{4}, \mathrm{C}_{2} \mathrm{H}_{6}$ and $\mathrm{C}_{2} \mathrm{H}_{2}, \mathrm{CO}_{2}$ and $\mathrm{CO}$ gas concentrations are the input vectors for the network

Anfis uses a hybrid learning algorithm to identify parameters of Sugeno-type fuzzy inference systems. It applies a combination of the least-squares method and the back propagation gradient descent method for training FIS membership function parameters to emulate a given training data set. 


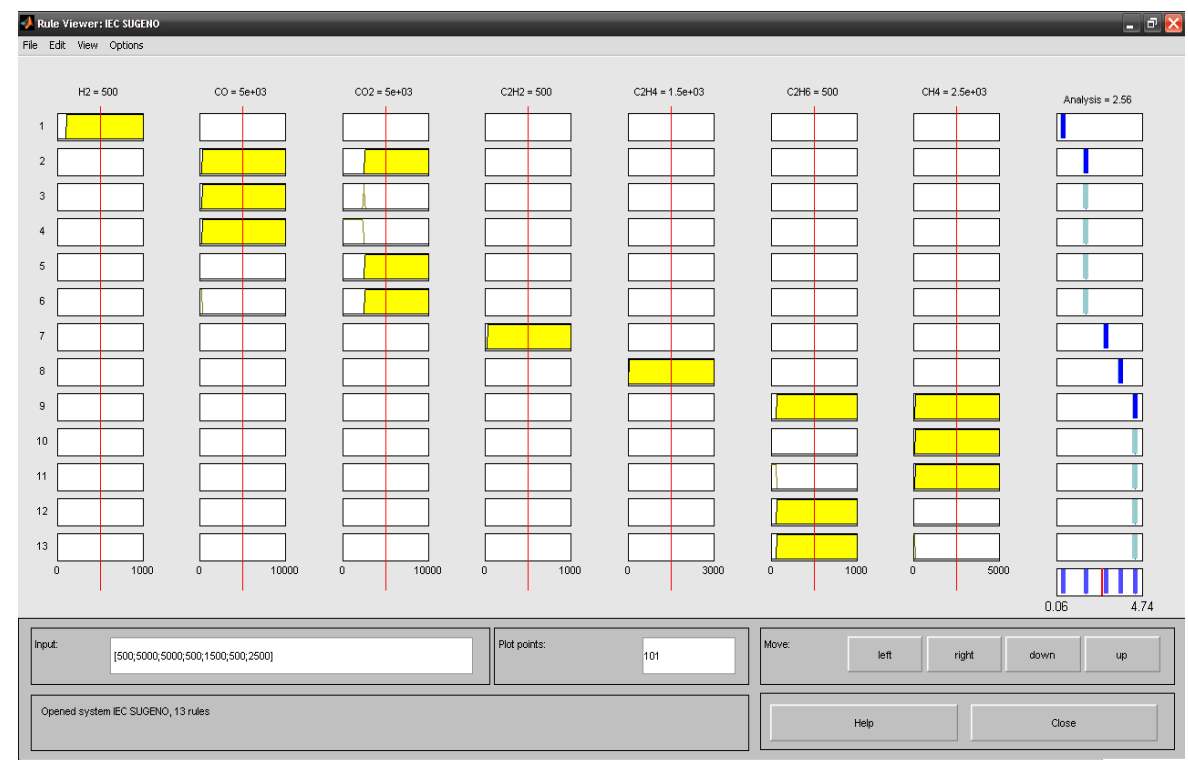

Figure (4) Rule base of Sugeno System to Model Adaptive Neuro Fuzzy Inference system

Anfis can also be invoked using an optional argument for model validation. Anfis only supports Sugeno-type systems. In this work a Sugeno type fuzzy system is initially created with $\mathrm{H}_{2}, \mathrm{CH}_{4}, \mathrm{C}_{2} \mathrm{H}_{4}, \mathrm{C}_{2} \mathrm{H}_{6}$ and $\mathrm{C}_{2} \mathrm{H}_{2}, \mathrm{CO} 2$ and $\mathrm{CO}$ gas concentrations as input vectors for the network.

Initially the system is trained using a data set which contains around 40 data inputs which has different types of faults and no faults condition represented by them. This data is essential in the generation and training of the ANFIS from the basic fuzzy structure. ANFIS has around 177 rules derived from the basic fuzzy structure. The ANFIS model structure that is generated for the analysis is presented in the below figure (5).

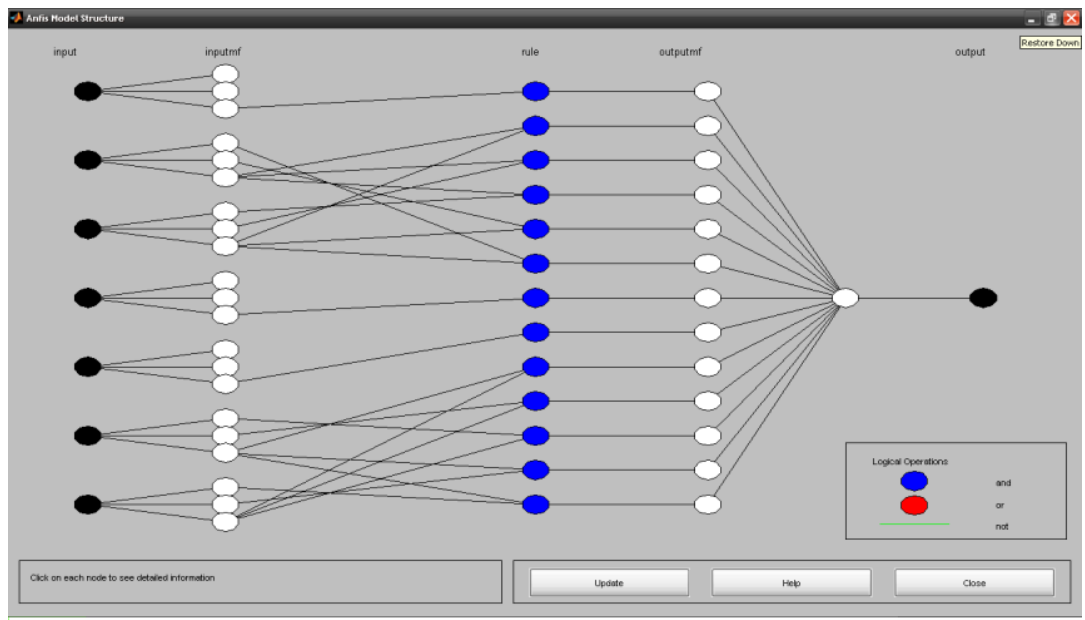

Figure (5) ANFIS Model Structure

The above ANFIS system which is conceptually based on KEY gas method is capable of identifying faults like Corona, Arcing, High Temperature Oil Break Down, Cellulose Insulation Break Down, etc.

\section{Results and Conclusion}

A comprehensive tool capable of performing different analysis as required by the user is designed. The tool is coded using Matlab Version 7.1 A Graphical User Interface is Designed for to enable the user to have seamless analysis of the data using different methods. Both Traditional Methods and methods based on fuzzy based analysis are available in the tool. 


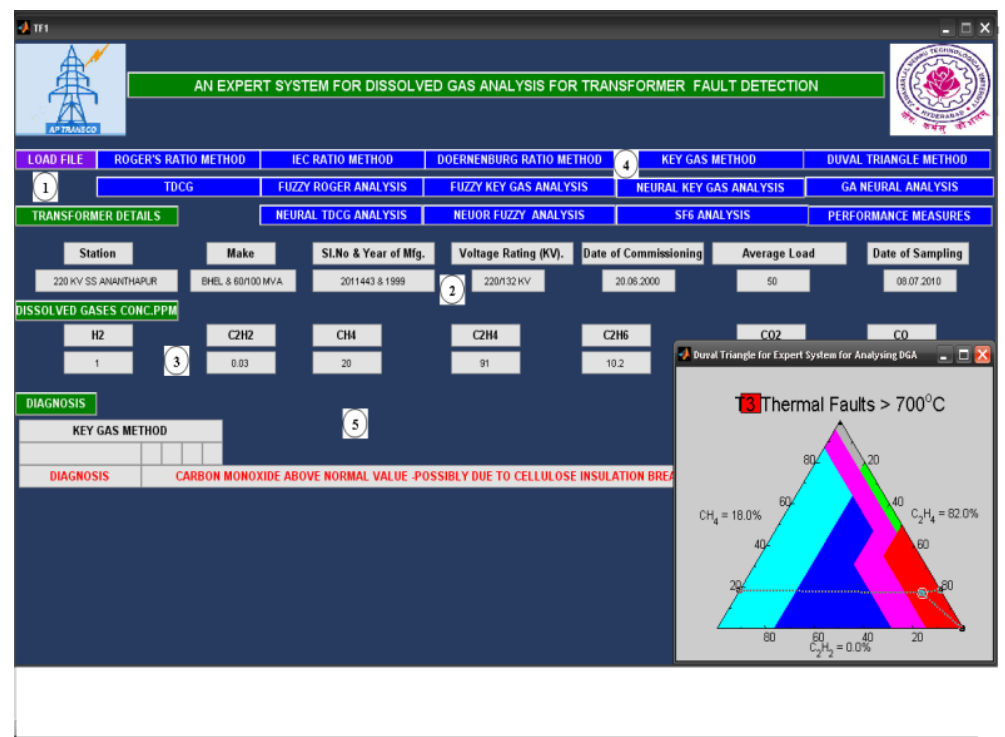

Figure (6): Snap Shot of Graphical User Interface

The data required for analysis is fed through an Excel sheet in predefined format. This helps in standardizing the input methods and helps in avoiding user induced error. In this work we have used the data format as used by APTRANSCO for collection of Dissolved Gas Analysis data. Once the Data is loaded the basic information about the transformer like its capacity, location, Make, average, load, date of commissioning are displayed in the GUI. Similarly the concentration of different gases in the sample under study is also depicted .Upon clicking the Load button the user is prompted to select a particular Excel work book and a specific sheet for analysis. Once the data is loaded, the user can select the method for analyzing the Data. The functional icons present in the GUI can be described as below in reference to the Figure (6)

1- Functional icon used to load the data for analysis through a Excel spread sheet

2- The Transformer location and other particulars like rating are displayed here.

3- The Concentration of dissolved gases being analyzed is displayed here

4- Functional icons used to execute different methods of analysis

5- Results of the diagnosis are displayed here.

Whenever the value of the gas being analyzed is in excess of stipulated value as specified by that method of analysis, the diagnosis information is depicted in 'RED' otherwise it is depicted in 'GREEN'. Alert Pop -Ups are also generated to warn about a specific Condition as depicted in Figure (6).

The Data that is used to validate the approaches discussed in this work is obtained from APTRANSCO. To validate our proposed approach we are considering data from 2 Substation of Kurnool and Ananthapur as Sample Cases like 220/KV Transformer - AP CARBIDES (KURNOOL) and 220/KV Transformer - SS Ananthapur. The analysis with respect to the NFIS system is primarily based on the values for Key Gas analysis. Based on the relation of fault gases, a decision can be made such as the presence of gas Acetylene which may indicate fault arcing if it is above certain limit in the insulation oil. In addition, the identification of Hydrogen in the presence of Methane may indicate corona or partial discharge. If corona developed into low energy sparking, a higher temperature is detected which lead to the additional presence of Acetylene. On the other hand, if sparking escalates to Arcing, the presence of Ethylene can also be detected. Furthermore, when Arcing takes place in the presence of cellulose, the high temperature deterioration of the solid insulation also releases carbon monoxide and carbon dioxide into the oil. The results of the proposed method are presented in the form of Table (1.0). The table consists of data of dissolved gases and the diagnosis provided by different methods. 
Table (1): Results of Analysis from the Proposed Tool

\begin{tabular}{|l|l|l|l|l|l|l|l|l|l|l|l|}
\hline H2 & CH4 & C2H4 & C2H6 & C2H2 & CO & CO2 & IEC & IEC & DUVAL & ANN & ANFIS \\
\hline 1.74 & 0.31 & 0.14 & 0.11 & 0.1 & 53.31 & 230.98 & 101 & DL & DL & CN & ARC \\
\hline 0.1 & 2.13 & 24.95 & 8.72 & 0.1 & 26.62 & 169.69 & 21 & $\begin{array}{l}\text { TF }-100- \\
200 C\end{array}$ & $\begin{array}{l}\text { TF }>700 \\
\text { C }\end{array}$ & CN & ARC \\
\hline 0.27 & 17.85 & 0.96 & 20.93 & 0.1 & 25.39 & 370.03 & - & - & TF<300C & ARC & ARC \\
\hline 145.33 & 14.11 & 6.11 & 4.69 & 0.1 & 646.8 & 5401.06 & - & - & DTF & CN & CN \\
\hline 10.11 & 16.26 & 26.46 & 3.96 & 0.1 & 1502.54 & 5275.27 & 22 & $\begin{array}{l}\text { TF-300- } \\
700 C\end{array}$ & $\begin{array}{l}\text { TF }>700 \\
\text { C }\end{array}$ & HTOB & CIB \\
\hline 13.2 & 5.05 & 52.18 & 16.81 & 0.1 & 339.29 & 1798.63 & - & - & $\begin{array}{l}\text { TF }>700 \\
\text { C }\end{array}$ & CN & ARC \\
\hline 3.74 & 0.6 & 0.78 & 0.22 & 0.1 & 24 & 303 & - & - & DTF & ARC & ARC \\
\hline 3.39 & 13.99 & 67.64 & 8.45 & 0 & 277.02 & 2879.82 & 22 & $\begin{array}{l}\text { TF-300- } \\
700 C\end{array}$ & $\begin{array}{l}\text { TF }>700 \\
\text { C }\end{array}$ & CN & CIB \\
\hline 0 & 14 & 13.6 & 5.2 & 0 & 602 & 2955 & 21 & $\begin{array}{l}\text { TF }-100- \\
200 C\end{array}$ & DT & CIB & CIB \\
\hline 0.0 & 2.1 & 13.6 & 1.1 & 0.0 & 176 & 1694 & 22 & $\begin{array}{l}\text { TF-300- } \\
700 C\end{array}$ & $\begin{array}{l}\text { TF }>700 \\
\text { C }\end{array}$ & CN & ARC \\
\hline 0.0 & 3.6 & 0.5 & 1.2 & 0.0 & 670 & 1397 & 20 & $\begin{array}{l}\text { TF-100- } \\
200 C\end{array}$ & $\begin{array}{l}\text { TF }<300 C \\
\text { CN }\end{array}$ & CIB \\
\hline 1.0 & 20.0 & 91.0 & 10.2 & 0.03 & 412 & 3437 & 22 & $\begin{array}{l}\text { TF-300- } \\
700 C\end{array}$ & $\begin{array}{l}\text { TF }>700 \\
\text { C }\end{array}$ & CN & HTOB \\
\hline 0.0 & 31 & 2.0 & 27.6 & 0.08 & 90 & 1605 & 20 & $\begin{array}{l}\text { TF-100- } \\
200 C\end{array}$ & $\begin{array}{l}\text { TF }<300 C \\
\text { CN }\end{array}$ & ARC \\
\hline 0 & 0.7 & 1.2 & 0 & 0 & 69 & 497 & - & - & DL & NF & NF \\
\hline 0 & 0.9 & 0.2 & 0 & 0 & 21 & 248 & - & - & DL & NF \\
\hline
\end{tabular}

TF- Thermal Faults; DL- Low Energy Discharge; DT- Thermal Discharge; CN-Corona ; ARC- Arcing ;CIB-Cellulose Insulation Break Down;HTOB-High Temperature Oil Break Down;NF-No Fault

According to the IEEE standard (C57.104-1991) [5], all the fault gases have their own norm value in normal and in faulty condition and the norm value varies due to different operating conditions, manufacturers and environmental factors such as humidity and weather. Due to this, different institutions from different countries have set their own sets of norm values in fault diagnosis. In this work, the IEEE norm value has been selected for Key Gas fault diagnostic method. It can be observed from the results that the ANFIS system is capable of identifying a wide range of faults in comparison with that of a pure ANN based diagnosis.

\section{References}

[1]. M. Duval and A. dePablo, "Interpretation of gas-in-oil analysis using new IEC publication 60599 and IEC TC 10 databases," IEEE Electrical Insulation Magazine, vol. 17, pp. 31-41, 2001.

[2]. M. Duval, "Dissolved gas analysis: It can save your transformer," IEEE Electrical Insulation Magazine, vol. 5, pp. $22-27,1989$.

[3]. IEEE Guide for the Interpretation of Gases Generated in Oil-Immersed Transformers, IEEE Std. C57.104-2008, 2009.

[4]. Mineral Oil-Impregnated Equipment in Service Guide to the Interpretation of Dissolved and Free Gases Analysis, IEC Std. Publication 60 599, 1999.

[5]. R. R. Rogers, "IEEE and IEC codes to interpret incipient faults in transformers, using gas in oil analysis," IEEE Transactions on Electrical Insulation, vol. EI-13, pp. 349-354, 1978.

[6]. J. J. Dukarm, "Tranformer oil diagnosis using fuzzy logic and neural networks," in CCECE/CCGEI, 1993, pp. 329-332.

[7]. Y. Zhang, X. Ding, Y. Liu, and P. Griffin, "An artificial neural network approach to transformer fault diagnosis," IEEE Transactions on Power Delivery, vol. 11, pp. 1836-1841, 1996.

[8]. Y.-C. Huang, H.-T. Yang, and C.-L. Huang, "Developing a new transformer fault diagnosis system through evolutionary fuzzy logic,” IEEE Transactions on Power Delivery, vol. 12, pp. 761-767, 1997.

[9]. H.-T. Yang and Y.-C. Huang, "Intelligent decision support for diagnosis of incipient transformer faults using self-organizing polynomial networks," IEEE Transactions on Power Delivery, vol. 13, pp. 946-952, 1998.

[10]. Z. Wang, Y. Liu, and P. J. Griffin, “A combined ann and expert system tool for transformer fault diagnosis," IEEE Transactions on Power Delivery, vol. 13, pp. 1224-1229, 1998.

[11]. J. Guardado, J. Naredo, P. Moreno, and C. Fuerte, "A comparative study of neural network efficiency in power transformers diagnosis using dissolved gas analysis," IEEE Transactions on Power Delivery, vol. 16, pp. 643-647, 2001.

[12]. Y.-C. Huang, "Evolving neural nets for fault diagnosis of power transformers,"IEEE Transactions on Power Delivery, vol. 18, pp. $843-848,2003$.

[13]. V. Miranda and A. R. G. Castro, "Improving the IEC table for transformer failure diagnosis with knowledge extraction from neural networks," IEEE Transactions on Power Delivery, vol. 20, pp. 2509-2516, 2005.

[14]. X. Hao and S. Cai-Xin, "Artificial immune network classification algorithm for fault diagnosis of power transformer," IEEE Transactions on Power Delivery, vol. 22, pp. 930-935, 2007

[15]. R. Naresh, V. Sharma, and M. Vashisth, "An integrated neural fuzzy approach for fault diagnosis of transformers," IEEE Transactions on Power Delivery, vol. 23, pp. 2017-2024, 2008. 
[16]. W. Chen, C. Pan, Y. Yun, and Y. Liu, "Wavelet networks in power transformers diagnosis using dissolved gas analysis," IEEE Transactions on Power Delivery, vol. 24, pp. 187-194, 2009.

[17]. A. Shintemirov, W. Tang, and Q. Wu, "Power transformer fault classification based on dissolved gas analysis by implementing bootstrap and genetic programming," IEEE Transactions on Systems, Man and Cybernetics, vol. 39, 2009.

[18]. Y.-C. Huang, "Evolving neural nets for fault diagnosis of power transformers," IEEE Trans. Power Del., vol. 18, no. 3, pp. 843848, July

[19]. Fuzzy Logic Toolbox, MATLAB

[20] Adaptive Neuro-Fuzzy Systems, Azar, Ahmad Taher, Electrical Communications \& Electronics Systems Engineering Department, Modern Science and Arts University (MSA),ISBN 978-953-7619-92-3,pp216 